DOI 10.37882/2223-2974.2021.03.19

\title{
ПРОБЛЕМЫ ОЦЕНОК РЕШЕНИЙ В ПРОЕКТНОМ УПРАВЛЕНИИ
}

\section{PROBLEMS OF EVALUATION \\ OF SOLUTIONS IN PROJECT MANAGEMENT}

\section{Melnichenko \\ O. Mikhnenko}

Summary: This article discusses issues related to the problems of evaluating solutions in project management at each stage of the life cycle of an innovative project, including the stage of development and use, from the point of view of both the project itself and from the point of view of the project portfolio and its effectiveness.

Keywords: project management, innovation, innovative project.
Мельниченко Вадим Николаевич

аспирант, Российский университет транспорта (МИИТ)

mvadimn94@mail.ru

Михненко Олег Евгеньевич

Ә.э.н., профессор, Российский университет транспорта

(МИИТ)

stat0243@yandex.ru

Аннотация: В данной статье рассматриваются вопросы, связанные с проблемами оценок решений в проектном управлении на каждой стадии жизненного цикла инновационного проекта, включая стадию освоения и использования, с точки зрения, как самого проекта, так и с точки зрения портфеля проектов и его эффективности.

Ключевые слова: проектное управление, инновации, инновационный проект.
И звестный афоризм: «нельзя управлять тем, что нельзя измерить», в своей реализации принимает форму, когда в целенаправленную деятельность встраивается аппарат измерения явлений и процессов. Функционирование этого аппарата обусловлено многим. Но в конечном итоге - одним: системой статистических показателей как основы построения информационной модели управляемого явления - процесса обеспечивая реализации принципа парности категорий "управленческое решение» + «информационная модель».

В такой системе определяющая роль отводится показателям - оценкам, построенным как меры критерия поведения-развития объективной реальности. Примером такой оценки выступают показатели эффективности функционирования производства, которые строятся исходя из критерия «максимум эффекта при минимуме используемых ресурсов», и, как следствие, имеющего меру в виде отношения «объем эффекта/объем примененного ресурса» или «объем эффекта/объем затраченных ресурсов».

Как можно видеть, все они - типичные представители данного класса показателей являются мерами сравнения показателя результата с показателем фактора, определяющего способность получения данного результата. Востребованность таких показателей-оценок в управлении обусловлена тем, что они являются мерой результативности функционирования самоуправляемой системы, которая дана нам в виде или возрастающей эффективности производства, если оценивается динамика показателя как таковая, или степени достижения цели, например, в повышении эффективности производства, когда достигнутый в процессе функционирования уровень эффективности сравнивается с целевым (чаще всего запланированном) уровнем, характеризующим возрастающую (рациональную, оптимальную) эффективность производства как цель функционирования. Поэтому не удивительно, что в проектном управлении, вообще, и в управлении проектами, в частности, показатели - оценки принимаемых решений и их исполнения играют роль ключевого инструмента. И как следствие, решения проблем их конструирования как показателей, отвечающих самым высоким требованиям предметности, точности и конкретности, методологии их исчисления в процессе наблюдения реальной действительности и методологии их использования в процессах принятия управленческих решений, занимают одно из центральных мест в организации и осуществлении управленческой деятельности [1,2]. При этом решение проблем в целях осуществления эффективного управления достигает поставленной цели, если в определенной степени является отражением принципов управления как такового.

Теория и практика имеет дело с определенным разнообразием методологий управления проектами. К числу наиболее продвинутых относят [3]:

- традиционную каскадную методологию, предпочтительную в проектах, результатом выполнения которых является материальный объект;

- методологию PRICE 2 (Projects in Controlled Enviroments), эффективную в крупных проектах, где крайне мала вероятность изменения объемов работ и требований к ним;

- методологию APG (Agile Project Managment), применяемую в случае, когда точное представление о том, каким должен быть конечный продукт и его жизненный цикл, формируется в процессе исполнения проекта; 
(Методология RAD (Rapid Application Development быстрая разработка приложений) является узко специализированной, так как используется в проектах, конечным продуктом которых выступают программные приложения в системах обработки информации).

При всех отличиях эти методологии объединяет подход, при котором управляемый проект рассматривается как многоэтапный процесс, где каждый этап связан с конкретной фазой жизненного цикла, представляет конкретный комплекс действий, связан с получением конкретного продукта-результата и имеет соответствующие зоны ответственности. Отличие их состоит в наборах конкретных принципов, конкретных аспектов и конкретных подходах к структуризации процесса. Последнее имеет сегодня определяющее значение, так как и теория и практика доказывают, что в организации и управлении производственной деятельностью эффективен процессо-ориентированный подход.

В наибольшей степени это присуще методологии PRICE 2. Причем она фокусируется на процессах, относящихся к высшему уровню (функциям) собственно управления как процессу принятия управленческого решения, организации его исполнения и соответствующего контроля.

В первую очередь обращает на себя внимание набор принципов этой методологии. Вот некоторые из основных.

Постоянная оченка экономической необходимости, когда неизменная экономическая выгода проекта на протяжении всего жизненного цикла рассматривается как системное свойство, определяющее функционирование элементов в составе целостной системы, коей выступает сам проект.

Создание ролевой модели, представляющей ясную организационную структуры всей системы управляемых процессов (процедур) в составе этапов и этапов в составе проекта с их ценностями и технологиями.

Управление по этапам, предполагающее планирование по этапам проекта, мониторинг и контроль каждого из них и проекта в целом.

Фокус на продукты, определяющий необходимость концентрироваться на определении и достижении качества продуктов - результатов этапа и проекта в целом.

Адаптация к среде проекта, согласно чему осуществляется адаптация процессов и управления проектом к требованиям внешней и внутренней среды, и как следствие, к изменяющимся масштабам работ, их сложности и квалификационным требованиям, и степени риска.
Что касается конкретных аспектов методологии управления проектом PRICE 2, которые находятся в центре внимания на протяжении всего жизненного цикла проекта, то к числу определяющих надо отнести следующие.

Обоснование проекта, когда акцент делается на установление и обеспечение получения ценности, которую проект приносит организации.

Организация, когда акцент делается на четком распределение роли и строгой ответственности между взаимосвязанными и взаимообусловленными процессами в составе этапов и между этапами в целях эффективного управления проектом в целом.

Качество, когда акцент делается на установление высоких требований к критерию и качеству проекта и определение порядка функционирования, который их обеспечит.

План, когда акцент делается на разработку планов для процессов и их комплексов различной степени общности и инструментов управления, обеспечивающие их (планов) выполнение

Риски, когда акцент делается на выстраивании механизмов управления, которые обеспечивают решение проблем, обусловленных наличием неопределенности в плане проекта и внешней среде.

Изменения, когда акцент делается на создание инструментов оценивания непредвиденных проблем (задач) и реализации способов реагирования на них.

Прогресс, когда акцент делается на превращение эффективной реализуемости процессов и этапов, высокого качества выполнения планов в факторы совершенствования и развития проекта.

Значимость представленных принципов и акцентов методологии управления определяется тем, что конструируемый показатель как элемент высокоразвитой системы будет играть свою роль, если он как качественно определенное количество не находится в противоречии с ними. Надо отметить, что сегодня мы сталкиваемся с положением, когда в управлении ставятся одни целевые задачи, а показатель отражает нечто, которое если и относится к целевой задаче, то во многом косвенно. Одновременно с этим любые действия, реализующие логико-математической аппарат преобразований в отношении показателей, не должны приводить к утрате их качественной определенности.

В настоящее время имеет место большое количество предложений по реализации конкретных концепций построения показателей-оценок для проектного управ- 
ления. Определяя отношение к ним, важно понять, что даже самое крупное преобразование, осуществляемое на принципах проектного управления, по существу является большой системой, элементами которой выступают проекты той или иной степени общности. В такой системе каждый элемент, с одной стороны, функционирует в соответствие положению в системе (связи и взаимоотношения с другими элементами) и реализует соответствующую функцию исходя из требований функции общей системы;

с другой стороны, выступает как относительно обособленный элемент, выполняющий свою функции с определенной степенью эффективности, причем эффективность функционирования элементов в их множестве определяет эффективность системы в целом;

с третьей стороны, и это очень важно, через управление его поведением осуществляется управление поведением большой системы, где само поведение элемента выступает как многофакторное явление и целенаправленно управляется через воздействие на факторы.

Как следует из вышесказанного, в проектном управлении определяющую роль играет конкретный проект в их множестве, а эффективность проектного управления будет определяться эффективностью управления проектами в их взаимосвязи и взаимообусловленности. Учитывая это, рассмотрим некоторые подходы к формированию систем оценок в управлении проектом.

Отметим, что проект может быть рассмотрен в узком и широком смысле. В узком смысле проект, согласно международным стандартам, рассматривается как временное предприятие (предпринятие), направленное на создание уникального продукта в соответствие с заданными ресурсами и требованиями. Откуда следует, что проект ограничен во времени, а это является его основной характеристикой как мероприятия, связанного со сдачей продукта заказчику. В другом случае границы проекта расширяются за счет процесса использования по своему назначению созданного продукта. Такое расширенное толкование обусловлено несколькими обстоятельствами, где важное, но не единственное - это то, что продукт (результат) проекта предназначен для применения/использования/потребления. Поэтому качество решений управленческих задач в рамках управления проектом проявляются на стадии использования продукта. На это обращается внимание, например в [4]. Более того, сама эффективность проекта, результатом которого является продукт - конкретная потребительная стоимость, проявляется именно в процессе его потребления / использования.

Учитывая это, жизненный цикл проекта будем рассматривать как целостный процесс со структурой, включающей следующие стадии: разработка концепции проекта, подтверждение концепции проекта, проектирование и прикладные исследования, производство новых образ- цов и пилотные продажи, наращивание производства и борьба за долю на рынках, стабилизация производства и получение прибыли [5].

Разработка концепции проекта предопределяет, что конкретная новация, доведенная до состояния инновации, внедряемой в производство (в широком смысле), подлежит оценке с позиций эффективности. Поскольку не все параметры процессов создания и использования инновационного продукта задаются точно, то оценки эффективности рассматриваются как предпроектные. Однако это означает, что они могут быть определены в относительно высокой степени неопределенности, которая в последующем становится источником различных рисков. И как следствие, к вопросам предпроектных оценок должны предъявляться достаточно высокие требования и, в первую очередь с точки зрения методологии их получения.

В работах, касающихся проблем оценки решений в области инноваций, нами подчеркивался тот факт, что такое решение должно оцениваться с позиций нескольких критериев эффективности, где экономическая - один из многих. В частности, если рассматривается проект, связанный с инновациями в области транспортных систем, связанных с перевозками грузов, то речь должна идти об оценках следующих, объективно дополняющими друг друга явлений [6,7.8]. Здесь социальная эффективность определяется способностью транспорта удовлетворять потребности в перевозках в требуемых объемах и надлежащего качества. Экономическую эффективность определяет способность транспорта в складывающихся общеэкономических условиях произвести продукцию и получить доход от оказания услуг грузового транспорта в объеме, позволяющем покрыть затраты на перевозки и иметь сбережения. Технологическая эффективность означает способность транспорта реализовать технологический процесс транспортировки с наименьшими затратами ресурсов во всем их разнообразии. Техническую эффективность надо связывать со способностью транспорта содержать техническую базу перевозочного процесса в размере, обеспечивающем в полном объеме осуществление перевозочного процесса в порядке удовлетворения потребностей в транспортировке грузов с соблюдением требований к качеству транспортного обслуживания. Объединяющим все названные аспекты эффективности производства выступает организационная эффективность, как способности обеспечить наивысший уровень надежности функционирования элементов и транспортной системы в целом.

При этом значимость соответствующей оценки в их множестве исходит из того, что между указанными аспектами эффективности объективно устанавливаются отношения иерархии, где высший уровень занимает социальная эффективность в различных ее проявлени- 
ях, включая, в том числе экологические. Согласно этому любое решение с позиций конкретных критериев будет признаваться эффективным, если оно одновременно эффективно и в точки зрения социальной эффективности, по меньшей мере, не ухудшая ее показатели.

Подтверждение концепции проекта как стадия проекта основана на показателях ожидаемых инвестиционных затрат и оценках ожидаемой эффективности инвестиций. Здесь эффективность проекта рассматривается с позиций мероприятия, в котором имеют место затраты, покрытие которых осуществляется за счет доходов от применения / использования инновации в производстве. При этом важно учитывать, что управленческое решение связано с выбором решения из множества вариантов, представленными конкретными факторами стоимости затрат и эффективности инновации, где стоимость проекта в большей степени определяется выбором конкретной технологии реализации конкретного технического решения, с одной стороны, а само техническое решение имеет определенный уровень параметров экономического эффекта. Поэтому уже здесь используются показатели-оценки, основанные на одновременном учете эффекта и затрат: чистого денежного потока, чистой добавленной стоимости, чистой или валовой прибыли и соответствующих показателей эффективности проекта на основе сопоставления эффектов и затрат.

Для оценки управленческих решений на данной стадии инновационного проекта важно понимание, что сами оценки определяются как ожидаемые, и, как следствие, содержат элемент неопределенности. Чтобы повысить степень надежности принимаемых решений, необходим учет тех рисков, которые с наибольшей вероятностью возникнут в ходе реализации проектов в силу изменений, как во внешней, так и во внутренней бизнессреде. Здесь в управлении проектами приходится сталкиваться с рисками текущей реализации проекта как мероприятия и рисками стратегического развития, В первую очередь организации, внедряющей инновацию, как продукт проекта. Поэтому востребованными становятся разработки по совершенствованию методик расчетов показателей затрат и доходов, в наибольшей степени учитывающие подобные риски. Однако на данной стадии жизненного цикла проекта актуальной становится проблема учета рисков стратегического развития.

Проектирование и прикладные исследования связано с разработкой целостных комплексов технологических процессов получения результата с определенными техническими (конструктивными) параметрами, с одной стороны, и определения порядка финансирования при осуществлении разрабатываемых комплексов. Оставляя В стороне организацию и управление собственно процессом разработки технологических и иных решений, отметим, в самом процессе обязательно присутствуют эле- менты поиска наилучших вариантов решений, которые и становятся объектом оценки. При этом важно учитывать, что конкретное технологическое решение относится к комплексу выполняемых взаимообусловленных работ, каждая из которых характеризуется соответствующими параметрами затрат ресурсов в их многообразии: труд, техника, материалы и энергия и т.д. И как следствие, проект получает оценку с точки зрения затрат, но в отличие от ожидаемых, предпроектных они характеризуются, во-первых, выбором конкретных технологий выполнения работ в их согласованности по объемам и времени, во-вторых, распределением затрат по их видам с требуемой детализацией, и в-третьих, распределением затрат во времени с необходимой подробностью.

Не меньшим разнообразием отличаются варианты в части организации финансирования проекта. Эффективность инвестиций как финансовых ресурсов на покрытие затрат определяется различием схем финансирования, представляющих, с одной стороны, конкретные источники финансирования: собственные накопления организации, заемные средства у финансовых посредников (займы и кредиты), средства сторонних участников проекта, средства государственного бюджета, а с другой стороны, порядок финансирования: единовременные поступления, распределенное во времени финансирование. И здесь возникает проблема оценки варианта решения с точки зрения участников проекта, а именно, исполнителя проекта с одной стороны и инвестора, финансирующего проект. Причем для последнего при принятии решений в первую очередь важна оценка уровня эффективности инвестиций.

Важно отметить, что к настоящему времени создан достаточно мощный теоретический и методологический задел, в том числе и виде разработанной системы показателей-оценок эффективности инвестиционных проектов. При его использовании в практической работе основные трудности связаны с необходимостью учета рисков, которые с наибольшей вероятностью возникнут в ходе реализации проектов из-за изменений факторов внешней и внутренней бизнес-среды. Причем в отличие от выше рассматриваемых стратегических рисков на данное стадии жизненного цикла к учету принимаются только те, которые могут возникать в процессе реализации/осуществления проекта и квалифицируются как текущие.

Производство новых образцов и пилотные продажи в случае транспортного производства в конечном итоге следует связывать с производством нового инновационного продукта, требующего реализацию инновационной технологии на базе инновационных образцов сооружений, подвижного состава, иных машин и оборудования.

Иллюстрацией этого служит продукт в виде высоко- 
скоростных пассажирских перевозок, для осуществления которых на данной стадии жизненного цикла проекта в процессе строительства создается инновационная инфраструктура, в том числе в части путевого и энергетического хозяйства, в транспортном машиностроении создается производственная база и организуется производство инновационного подвижного состава, в рамках транспортной организации разрабатывается инновационная технология перевозочного процесса в части организации обращения высокоскоростных поездов и т.п. И жизнедеятельность такого проекта будут определять пилотные перевозки на ограниченных полигонах сети в объемах, позволяющих доказать приемлемый уровень потребительских свойств таких перевозок, в том числе с точки зрения эксплуатационных затрат и стоимости поездки, с одной стороны, и высокий уровень потребительных свойств в части сокращения времени поездки в условиях повышенного комфорта, с другой стороны.

В дополнение к рассмотренному не исключено ограничение продукта проекта в виде использованием новых образцов техники в действующих технологиях перевозочного процесса, или новых технологий в производстве традиционного продукта - будь то перевозки традиционных грузов или перевозки пассажиров в традиционных сообщениях.

Управленческие решения связаны с первую очередь с производством комплекса работ в их взаимосвязи и взаимообусловленности с соблюдением всех требований к качеству работ и затрат ресурсов. Организуя эту деятельность как систему бизнес-процессов, реализуются принципы бюджетного управления. При этом выявляются и компенсируются возникающие риски в настоящем и возможные в будущем, с одной стороны, и выявляются, и используются факторы, способствующие оптимизации стоимости проекта в данной стадии и повышения потребительной стоимости - полезности продукта бизнес-процессов и проекта в целом, с другой.

И как следствие, оценка решений в части управления через бюджет проекта строится на основе показателей фактической стоимость работ - продуктов бизнес-процессов, анализируемой с целью определения эффективности /неэффективности затрат;

оценка в управления рисками стоится на основе показателей вероятности наступления соответствующих событий на уровне элементарного и использования их в сценарном анализе для изучения последствий на уровне поведения общего той или иной степени общности;

оценки мероприятий по оптимизации стоимости проекта на основе показателей снижения стоимости (затрат) на создание продукта бизнес-процесса или проекта в целом в расчете на единицу эффекта;

оценки мероприятий по повышению полезности продукта на основе показателей возрастающего эффекта в сравнении с затратами на его получения, с одной стороны, и показателей возрастающей конкурентоспособности продукта с выходом на оценки возрастающего спроса на продукт, с другой.

В целом эффективность управления проектом на данной стадии жизненного цикла может быть оценена на основе показателя фактической эффективности инвестиционного проекта, когда фактическая стоимость проекта в виде общего объема фактических инвестиций сопоставляется с размерами эффекта в виде ожидаемого объема удовлетворяемого спроса на результат проекта и связанным с ним объемом доходов в виде ожидаемой выручки, добавленной стоимости и прибыли.

Наращивание производства и борьба за долю на рынках представляет ту стадию жизненного цикла, которая связана с применением/использованием продукта проекта в отличие от предшествующих стадий, связанных с созданием продукта инновационного проекта. В случае нашего примера речь идет о начальном этапе широкомасштабного внедрения в повседневную жизнь населения высокоскоростных пассажирских перевозок. В [4] показано, что в едином процессе использование инновационного продукта следует различать такие этапы как этап запуска новации, достигая пика нередко завышенных ожиданий; этап неоправданных надежд, опускаясь на дно впадины разочарования; этап aдanmaции к новации, завершающегося признанием реальной полезности; этап востребованной продуктивности вследствие общепризнанной стабильной применяемости. Такое понимание динамики востребованности используемого продукта проекта, формируемой за пределами организации, должно быть заложено в основу управления на данном этапе жизненного цикла. Это означает, что управляется эффективность процесса освоения объемов деятельности, распределенных в границах временного интервала неравномерно в соответствие указанной закономерности. Тогда показатели-оценки будут отражать, с одной стороны, ускорение или замедление процесса выхода на уровень стабильного производства, с другой стороны, эффективность собственно деятельности, учитывая, что в производстве фактически получаемого эффекта применены/затрачены ресурсы в определенных объемах.

В такой текущей деятельности как системе бизнеспроцессов оправдано использование инструментов бюджетного управления. И как следствие, востребованы показатели-оценки исполнения бюджетов, исчисляемые исходя из следующего. Исполнение бюджета есть многофакторное явление, когда в большом числе разнородных факторов по существу будут присутствовать те, которые оказывают позитивное или негативное влияние с большей или меньшей силой.

Стабилизация прочзводства и получение прибы- 
ли - это тот этап жизненного цикла проекта, на котором реализуется цель проекта внедрения в производства инновации в виде осуществления полномасштабного использование продукта проекта. Поэтому на данном этапе возникают условия наиболее точной оценки его эффективности. А для этого становится востребованным, по меньшей мере, то множество показателей-оценок эффективности, которые использовались при решении вопросов осуществления проекта. Однако в отличие от этапов разработки концепции и подтверждения концепции проекта они представляют фактическую деятельность, что позволяет реализовать один из аспектов эффективного поведения, когда эффективность/результативность проекта оценивается исходя из категории «степень достижения цели - реализовать проект с заявленными параметрами эффективности».

Обозначенные подходы к оцениванию этапов проект и проекта в целом, а главное показатели-оценки определяются исходя из сущности внедряемой инновации. В условиях, когда развитие организации на принципах проектного управления предопределяет реализацию портфеля инновационных проектов, возникает проблема оценки множества проектов в составе портфеля, но исходя уже из сущности портфеля, которая отражается в показателях-оценках его эффективности.

Формирование подобного портфеля, как отображение принципов инновационного развития организации, в конечном итоге приводит к тому, что сам портфель выступает или как системный объект управления, в силу необходимо внедрения множества взаимосвязанных и взаимообусловленных инноваций в рамках определенной их (инноваций) системы, или аддитивный объект управления, который представляет собой объективно обусловленное множество независимых инноваций [9].

Когда конкретный проект рассматривается как относительно обособленный элемент в составе системного объекта, кроме оценок по существу инновации он - один из множества может и должен получить оценку как элемент целостной системы. Причем такая задача решается в условиях, когда эффект/результат системы не сводится к простой сумме эффектов проектов, входящих в ее состав. Методология получения таких оценок, основанная на положении, что эффективность поведения элемента определяет эффективность поведения системы в целом, представлена в $[9,10]$. Таким образом формируются условия, когда каждый отличный по существу инновации проект получает одинаковую оценку - вклад в эффективность инновационного портфеля, и как следствие формировании квалифицированных оценок проектов в их множестве как результат сравнительный анализ проектов между собой.
В случае портфеля как аддитивного объекта управления проблемы оценки инновационного проекта и портфеля в целом возникают в силу следующего. Каждый проект с присущей ему индивидуальностью вносит свой вклад в эффективность портфеля, причем эффект портфеля есть сумма эффектов формирующих его единиц. Но при этом сами эффекты проекта формируют совокупность несоизмеримых элементов. Общую меру такой совокупности можно получит на основе того единства, которое присуще каждой единице. Учитывая, что целью инновационного развития является повышение эффективности организации, такое единство может выражаться в том, что каждый проект вносит свой вклад в повышение измеряемой «рыночной стоимости компании» [5]. Тогда эффективность проекта и портфеля в целом будет определяться исходя из критерия «максимум эффекта при минимуме ресурсов», когда размеры эффекта и ресурсов портфеля есть суммы, соответственно, эффектов и ресурсов его элементов.

Как можно видеть единообразие методики оценки эффективности проектов в их множестве и портфеля в целом обеспечивает сравнение проектов между собой и каждого из них в составе портфеля. В последнем случае сравнение исходит из понимания того, что величина эффективности портфеля представляет собой среднюю из вариантов, в качестве которых выступают значения эффективности проектов в их множестве.

Значимость показателей-оценок как качественно определенных количеств в управлении проектами определяется тем, что реализация управленческих решений направлено на изменение в порядке реализации инновационных проектов количественным параметров при сохранении качественной определенности деятельности. Само принятие эффективного управленческого решения возможно при наличии знаний в виде ответов на вопросы:

- что произошло в виде количественных изменений, которые можно измерить?

- почему произошли такие, не иные количественные изменения в условиях действия большого числа факторов?

- в силу каких закономерностей имеют место изменения с данной интенсивностью?

- что может произойти в будущем в параметрах деятельности, в том числе при реализации планируемых конкретных мероприятий?

Но при этом очень важно, чтобы показатели - оценки конкретных проектов и инновационных портфелей были адекватными мерами реальной действительности в части поведения управляемых объектов по существу и организации управления. 


\section{ЛИТЕРАТУРА}

1. Михненко 0.Е. Проблемы управления экономическими процессами на железнодорожном транспорте: информационный аспект. - М.: МИИТ, 2001. 200 c.;

2. Михненко 0.Е. Цифровые технологии и эффективность статистических показателей /Цифровая трансформация в экономике транспортного комплекса: материалы международной научно-практической конференции/под ред. Соколова Ю.И.. Бубновой Г.В.. Каргиной Л.А.. Епишкина И.А.. - М.: Рут (МИИТ). 2019. - C.207- 216;

3. Мазур И.И., Шапиро В.Д., Ольдерогге Н.Г. Управление проектамибУчебное пособие /Под общ. Ред. И.И.Мазура. 2-е изд.. - М.: Омега - Л. 2005. -664 с.; Ньюэлл М. Управление проектами для профессионал.РуководствоУправление пректами

4. Мельниченко В.Н, Чикаревский В.С., Михненко О.Е. Инновация как объект проектного управления / Цифровая трансформация в экономике транспортного комплекса: материалы международной научно-практической конференции/под ред. Соколова Ю.И.. Бубновой Г.В.. Каргиной Л.А.. Епишкина И.А.. М.: РУТ (МИИТ). 2020. - C.00- 00

5. Ильин В.В., Михненко 0.Е., К вопросу эффективности реформирование организационных структур производственной деятельности /Вклад транспорта в экономическую безопасность //Материалы международной научно-практической конференции. - М.: МИИТ. 2017. -С.00-00

6. Михненко 0.Е., Ильин В. Какой железнодорожный транспорт обеспечит экономическую безопасность национальной экономики /Вклад транспорта в экономическую безопасность //Материалы международной научно-практической конференции. - М.: МИИТ. 2019. - С.00-00

7. Михненко О.Е, В.В. Ильин Эффекты мероприятий по безопасности движения поездов / Безопасность движения поездов// Труды Семнадцатой научнопрактической конференции. - М.: МГУПС (МИИТ), 2016, C.V-77 - V-80;

8. Михненко 0.Е. Информационные модели в управлении экономическими явлениями. - М.: МИИТ,2009. - 48с.;

9. Михненко 0.Е Вопросы анализа экономических явлений на основе статистической информации: Учебное пособие - М.: МИИТ.2007. - 98с.;

( ) Мельниченко Вадим Николаевич (mvadimn94@mail.ru), Михненко Олег Евгеньевич (stat0243@yandex.ru).

Журнал «Современная наука: актуальные проблемы теории и практики»

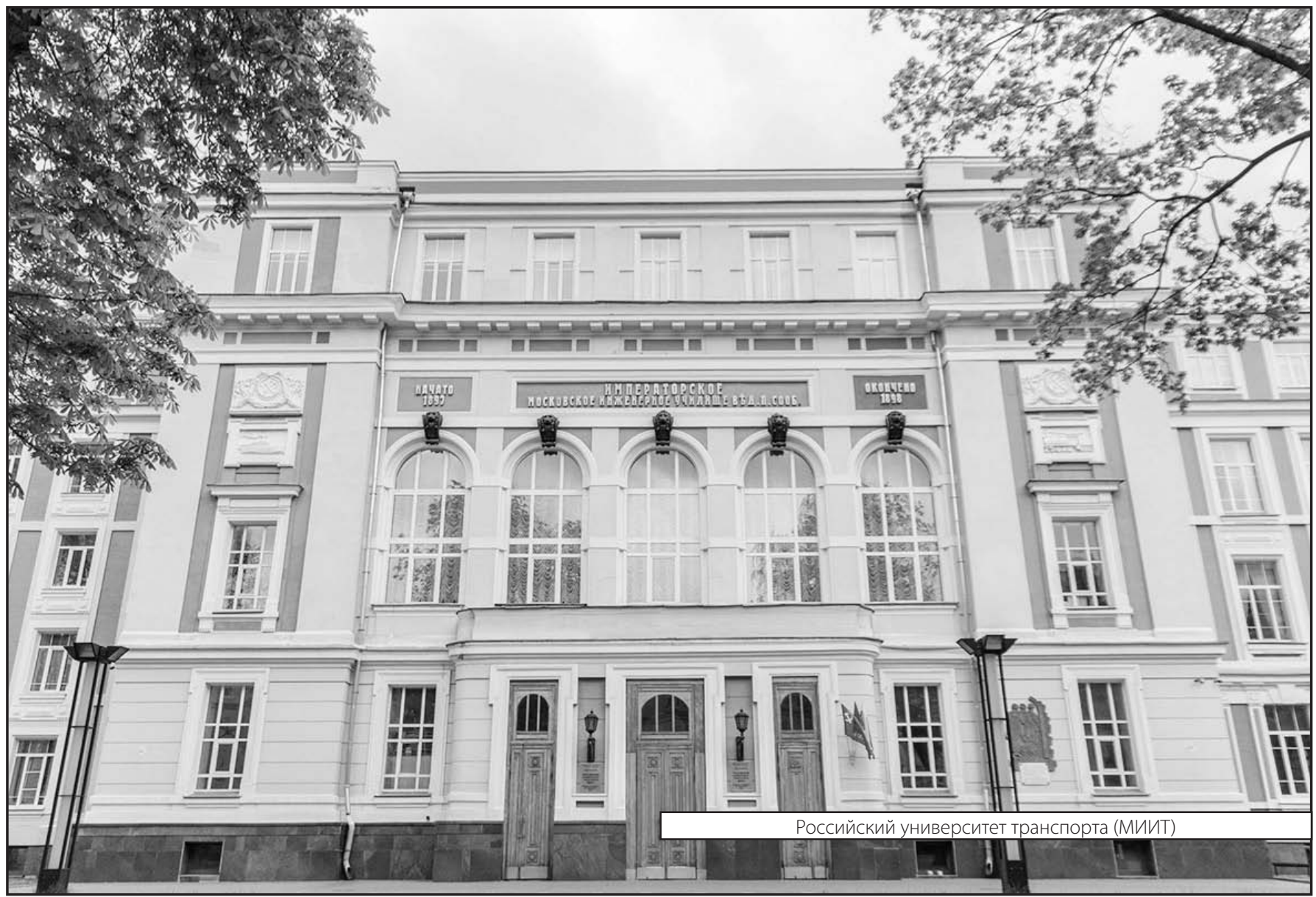

